\title{
Electromagnetic Expansion and Fragmentation of Hollow Aluminium 5052 Tube
}

\author{
Hitesh Choudhary ${ }^{1,2}$, Chiradeep Gupta ${ }^{3}$, Nirupama Tiwari ${ }^{4}$, Tanmay Kolge $^{2}$, Rajeev Kapoor ${ }^{3}$, \\ Archana Sharma ${ }^{2}$
}

\author{
${ }^{1}$ Homi Bhabha National Institute, Anushaktinagar, Mumbai, India \\ ${ }^{2}$ Accelerator \& Pulse Power Division, Bhabha Atomic Research Centre, Mumbai, India \\ ${ }^{3}$ Mechanical Metallurgy Division, Bhabha Atomic Research Centre, Mumbai, India \\ ${ }^{4}$ Laser \& Plasma Technology Division, Bhabha Atomic Research Centre, Mumbai, India \\ Email: hiteshchoudhary29@gmail.com
}

How to cite this paper: Choudhary, H., Gupta, C., Tiwari, N., Kolge, T., Kapoor, R. and Sharma, A. (2020) Electromagnetic Expansion and Fragmentation of Hollow Aluminium 5052 Tube. Journal of Minerals and Materials Characterization and Engineering, 8, 421-439.

https://doi.org/10.4236/jmmce.2020.86027

Received: August 15, 2020

Accepted: October 24, 2020

Published: October 27, 2020

Copyright $\odot 2020$ by author(s) and Scientific Research Publishing Inc. This work is licensed under the Creative Commons Attribution International License (CC BY 4.0).

http://creativecommons.org/licenses/by/4.0/

\begin{abstract}
Electromagnetic forming is a high-speed forming technology by which hollow profiles can be compressed or expanded. It is done with a pulsed magnetic field to apply Lorentz' forces at electrically conductive material. Electromagnetic hollow tube expansion is limited by the fragmentation tendency. This work attempts to use a combination of analytical and computational approach to compute the net tangential stress during tube expansion. A simplified analytical framework to estimate the temporal evolution of plastic stresses present in aluminium alloy AA5052 at low and high applied magnetic pressures is developed based upon dynamic imaging. The time resolved images captured using current synchronised high speed camera record the overall dimensional changes of the tube that is validated by multi-physics simulation of expansion process. Imaging of hollow tube expansions at two selected peak currents has been carried out at various current levels in the range 76 $160 \mathrm{kA}$. The direct visualisation of the increase in the tube diameter at two current levels provided a comparison of the developing net tangential stresses in the hollow tube during the undamaged and fragmented expansion. Imaging of tube expansion also facilitates the estimation of the strain rate experienced by the tube and was in the range of $\sim 1700 \mathrm{~s}^{-1}$ to $\sim 1200 \mathrm{~s}^{-1}$. The propensity of fragmentation was found to be due to the level and duration of generated tangential stresses above the yield stress during expansion of the aluminium tubes. Presented study provides a mean of exploiting the enhanced formability of aluminium alloys using electromagnetic forming.
\end{abstract}

\section{Keywords}

Electromagnetic Forming, Aluminium Alloys, High-Speed Imaging 


\section{Introduction}

Electromagnetic forming is realised by the interaction of pulsed magnetic field arising from a suitably placed pulsed solenoid coil which induces a secondary current in the conductive work-piece [1] [2]. As a result, a radial force on the work-piece originates which may have sufficient magnitude to deform it at high strain rates. This principle has been harnessed for non-contact expansion of metallic objects as well as for joining of dissimilar metals under the action of an electromagnetic pulse. The method is quite attractive for large sheet metal forming as it combines good quality with low production cost for manufacture of thin components [2].

Hollow tube and rings expansion under electromagnetic loading is the simplest form of the electromagnetic forming methods. A cylindrical coil is used to provide the electromagnetic force on the job piece tube surface. It has been shown that the profile of electromagnetic force generated by the cylindrical coil is maximum at its mid length. As a result, the tube deformation is in-homogenous in the axial direction [3]. This could cause strain incompatibilities of the deforming tube, thereby limiting the full extent of expansion without defects. Optimum coil design [2] or using field shapers [1] are some of the methods used for obtaining a more homogenous magnetic field force profile. In order to understand the fragmentation of the tubes, insights from the large body of experimental and theoretical simulation of the ring expansion process, emphasising necking formation, is found to be useful [3]. Primarily the emphasis is to provide an explanation for the delay in necking which some studies attribute to the strain rate sensitivity of the material such as aluminium [3] and others to inertial effects [3]. Unlike the situation of ring expansion, in case of unconstrained tube expansion the curvature of the expanding surface is non-trivial and hence needs to be modelled explicitly. With advancement in the availability of multi-physics simulation softwares, few recent simulations of the tube expansion process have been reported in literature [4] [5] [6] [7]. To the knowledge of the authors, the fragmentation behaviour of tubes has not been modelled. Thomas and Triantafyllidis [8] have studied the fragmentation of tubes by generating theoretical formability limits and experimentally comparing the multiaxial strain conditions near and far from the necked region. In order to understand the fragmentation of electromagnetic tubes, it is required to have a first-hand assessment of the stresses induced during the expansion process. Experimentally this approach could be addressed by directly recording the expansion by dynamic imaging.

The electromagnetic forming process occurs within few hundred micro-seconds, and hence the visualisation of the process becomes very challenging. Visualisation of the work-pieces would enable not only recording their relative motion but also estimate the parameters of deformation such as strain rate and stresses. Deformation processes in techniques such as impact Taylor test [9], plate on plate impact test [10] and split Hopkinson bar [11] occur in similar time frame 
as electromagnetic forming. In these techniques, dynamic behaviour of the objects has been investigated by the use of high-speed camera. For example, a ring expansion process captured by high-speed camera enabled the velocity of expansion as well as the nucleation of cracks along the circumference. Apart from recording the deformation of the object, the use of high-speed camera has also been to record the rapid dynamics of anode arc root in a dc arc plasma torch [12], single wire arc spray process [13] and collisions of aluminium plates occurring in magnetic pulse welding [14].

Aluminium-magnesium alloy AA5052 (Al-2.5Mg) - a commonly used alloy due to its excellent high strength to weight ratio, corrosion resistance, weldability and recycling potential-is a potential alloy for electromagnetic pulse welding. In this work electromagnetic free expansion of aluminium alloy AA 5052 tubes was carried out at different coil currents with the aim of providing reasonable estimates of the strain rate and stresses during expansion and fragmentation of the tubes. This was achieved by using a high-speed camera synchronised with the current signal to record the tube expansion.

\section{Experimental Methods}

\subsection{Electromagnetic Forming Set Up}

A tubular work-piece was formed using solenoid pulsed electromagnet in an electromagnetic forming set-up. The overall electrical circuit for the tube expansion is of capacitive-resistive-inductive nature as shown in Figure 1. In electromagnetic forming a capacitor bank is charged through power supply to store electrostatic energy in it. This stored energy is discharged into the electromagnet with the help of high voltage-high current switch. This produces a current of a damped sinusoidal waveform through the electromagnet, which in turn generates a time varying pulsed magnetic field that induces eddy currents through the surface of the work piece (metallic tube). The magnetic field between coil and work piece, and the current through the work piece generates a repulsive force $\boldsymbol{J} \times \boldsymbol{B}$ in the outward radial direction causing the tube to expand. Furthermore, the applied magnetic pressure causes deformation of the work-piece only in areas close to the winding of the coil.

The experimental setup (Figure 2) consisted of an electromagnetic coil, a capacitor bank, a high-speed camera interfaced with a personal computer (PC) and a digital storage oscilloscope (DSO). These are briefly described below.

1) Electromagnetic coil: Electromagnet (EM) used in the experiment had 7 number of turns, an outer diameter of $60 \mathrm{~mm}$ (64 mm with insulation) and inner diameter of $47 \mathrm{~mm}$. The coil was distributed over a length of $47 \mathrm{~mm}$, with the conductor of the coil having a cross-section of $5 \mathrm{~mm} \times 8 \mathrm{~mm}$ and a gap of 2 $\mathrm{mm}$ between turns. The AA5052 tube (work-piece) was fitted on the EM reinforced coil concentrically as shown in Figure 3. The resistance and inductance of the EM coil were measured using an LCR meter and the values were found to be very close to the respective calculated values (Table 1). 


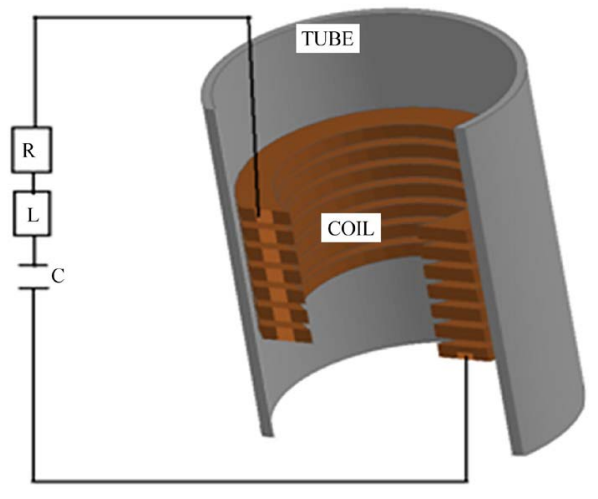

Figure 1. Schematic circuit showing the coil and the surrounding AA5052 tube used in electromagnetic forming experiment.

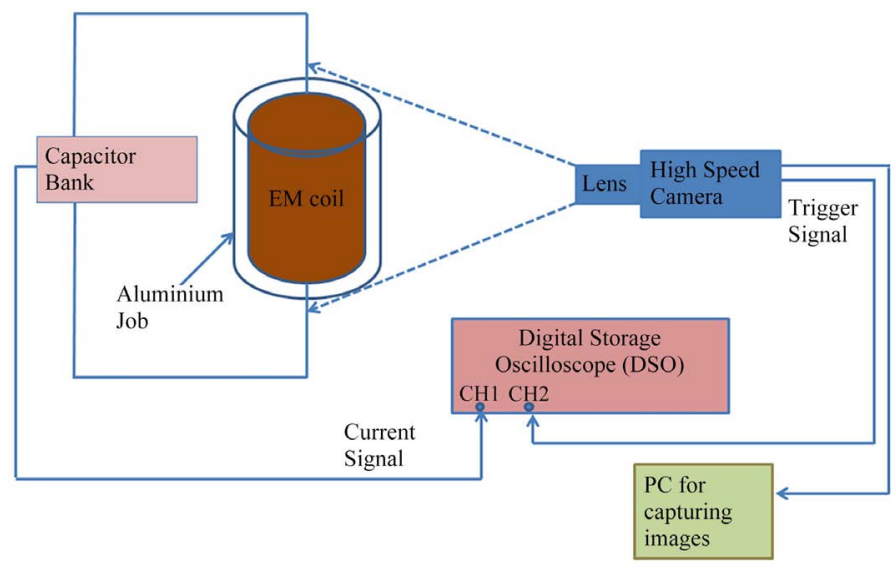

Figure 2. Schematic of the experimental setup used in the electromagnetic induced expansion of AA5052 tube.

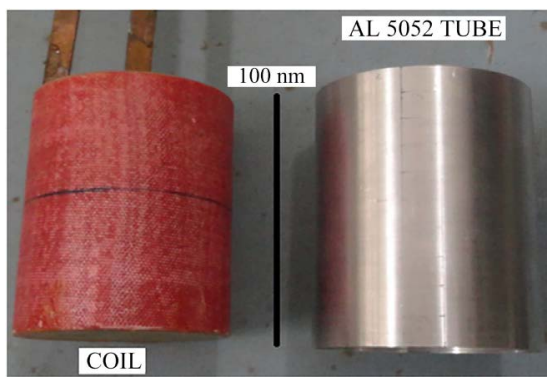

Figure 3. Photograph of the electromagnetic coil (left) and AA5052 tube (right).

Table 1. Calculated and measured parameters of pulsed magnet.

\begin{tabular}{|c|c|c|c|}
\hline & Parameters & Calculated & Measured using LCR meter \\
\hline 1 & $\begin{array}{l}\text { Resistance of the coil } \\
R=\frac{\rho}{f} \frac{N^{2}}{r_{1}} \frac{\pi}{2} \frac{(\alpha+1)}{\beta(\alpha-1)}\end{array}$ & $6.76 \mathrm{~m} \Omega$ & $6.1 \mathrm{~m} \Omega$ \\
\hline 2 & $\begin{array}{c}\text { Inductance of the coil } \\
L=\frac{\mu_{0} \pi}{8} r_{1} \frac{(\alpha+1)^{2}}{\beta} N^{2} g(\alpha, \beta)\end{array}$ & $1.77 \mu \mathrm{H}$ & $1.8 \mu \mathrm{H}$ \\
\hline
\end{tabular}


2) Capacitor Bank: A $224 \mu \mathrm{F}$ capacitor was used in the capacitor bank that can be charged up-to $20 \mathrm{kV}$ thereby capable of storing a maximum charging energy $\left(\frac{C V^{2}}{2}\right)$ of $44.8 \mathrm{~kJ}$, where the capacitance is $C$ and the charging voltage $V(t)$. In the experiments, the stored electrostatic energy in capacitor bank is discharged practically instantaneously into the electromagnetic coil by closing the spark gap switches (switch $S$ shown in Figure 4). The resulting current $i(t)$, is determined by the resistance $R$, inductance $L$ and capacitance $C$ of the circuit. The electrical equivalent circuit is shown in Figure 4. The expression of current through the coil is given by Equation (1). The expression of the resulting generation of magnetic pressure (in $\mathrm{MPa}$ ) is derived in Equation (2).

$$
\begin{gathered}
i(t)=\frac{V}{W_{d} L} \mathrm{e}^{-\in W_{n} t} \sin \left(W_{d} t\right) \\
B=\mu_{0} \frac{N I}{l} \\
P_{G e n}=\frac{B^{2}}{2 \mu_{o}}=\frac{\mu_{o} N^{2}}{2 l^{2}} i^{2}(t) ; \\
P_{\text {Gen }}=4.97 \frac{N^{2} V^{2}}{f^{2} L^{2}} \mathrm{e}^{-2 \in W_{n} t}\left(1-\cos \left(2 W_{d} t\right)\right),
\end{gathered}
$$

where, $V=$ charging voltage in $\mathrm{kV}, L=$ inductance in $\mu \mathrm{H}, R=$ resistance in $\Omega, C$ = capacitance in $\mu \mathrm{F}, W_{d}=$ damped frequency of oscillations, $f=\frac{W_{d}}{2 \pi}$ in $\mathrm{kHz}$, $W_{n}=$ natural frequency of oscillations, $\in=$ damping coefficient, $B=$ magnetic field in Tesla, $H=$ magnetic field intensity in AT/m, $l=$ coil's mean length, $i(t)=$ current in coil, $P=$ magnetic pressure in $\mathrm{MPa}, N=$ number of turns of the coil.

3) High-speed camera interfaced with $P C$ and digital storage oscilloscope $(D S O)$ : To capture electromagnetic expansion, a high-speed camera (Make: PCO AG, Germany, Model No. pco1200 hs, standard sensor size 1280 pixels $\times 1024$ pixels, frame rate 500 frames per second FPS) was used. As the expansion process is expected to be completed in few hundred microseconds $(\sim 500 \mu \mathrm{s})$ the number of frames per second of camera was set to 10,000 FPS, over a sensor area of 980 pixel $\times 60$ pixels focused on the tubular object. A flood light of $1 \mathrm{~kW}$
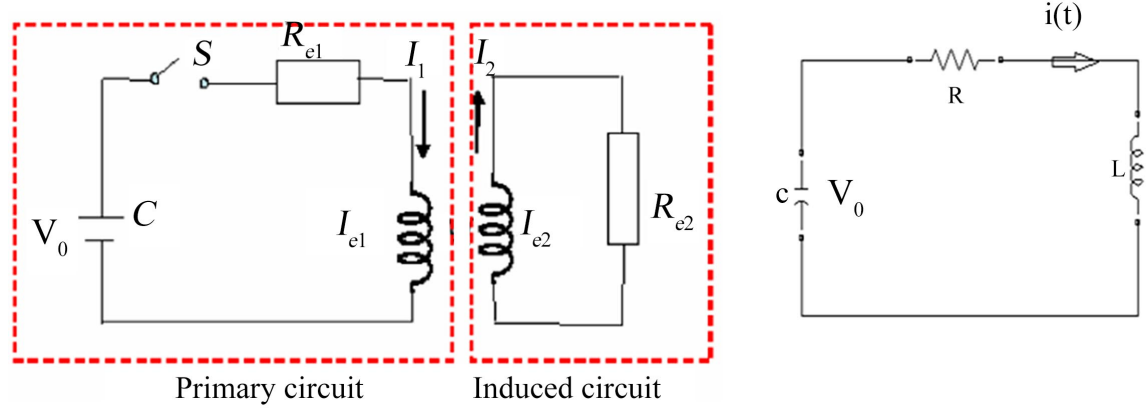

Figure 4. Electrical equivalent circuit of EMF (left) and referred to primary circuit (right). 
power was used to illuminate the object to reduce the exposure time. CAMWARE (provided by high-speed camera manufacturer) software was used for capturing images on PC. A trigger signal ( $5 \mathrm{~V}$ ) from camera was given to the oscilloscope. Current signal from capacitor bank was used to trigger the oscilloscope. Simultaneous acquisition of current signal from EM coil power supply and signal from camera was done using DSO in order to synchronize the captured images in the high-speed camera. Calibration for image size was done before capturing the images, with one pixel corresponding to $1 \mathrm{~mm}$.

\subsection{High Rate Testing}

Annealed AA5052 (the tube material) was tested using a split-Hokinson bar for determining the room temperature high strain rate uniaxial properties. For this purpose, cylindrical compression specimens of $5 \mathrm{~mm}$ diameter and $5 \mathrm{~mm}$ height were used. Multiple tests were carried out within the strain rate range of 1200 $3000 \mathrm{~s}^{-1}$. At these strain rates, the tests are adiabatic in nature. For details of the test and the equipment used see refs. [15] [16].

\subsection{Tube Material and Geometry}

The work-piece chosen for the studies was annealed AA5052 grade aluminium alloy with room temperature quasi-static yield strength of about 60 - $80 \mathrm{MPa}$. The tube subjected to electromagnetic loading was a hollow cylinder of inner diameter $65 \mathrm{~mm}$, outer diameter $70 \mathrm{~mm}$ and length $100 \mathrm{~mm}$.

\subsection{Electromagnetic Forming Experiments Carried out}

Ten electromagnetic forming experiments were carried out, each at different peak coil currents and achieving a specific frequency, as shown in Table 2. Note that two tests were carried out with peak coil current of 131, but with different frequencies.

Table 2. Experiments detail of EMF experiments carried out.

\begin{tabular}{ccc}
\hline Sl. No. & Peak Coil current in kA & Frequency kHz \\
\hline 1 & 76.5 & 13.33 \\
2 & 95 & 10.24 \\
3 & 101 & 10.24 \\
4 & 113 & 10.87 \\
5 & 117 & 10.87 \\
6 & 131 & 7.84 \\
7 & 131 & 10.6 \\
8 & 140 & 7.04 \\
9 & 149 & 7.04 \\
10 & 160 & 7.04 \\
\hline
\end{tabular}




\subsection{Analytical Framework for Estimation of Deformation Parameters}

Deformation parameters of circumferential stress, true strain and strain rate are computed from the time interleaved images by measuring the outer diameter of the tube as a function of time and estimating the velocity of expansion. The strain in the tube due to its radial expansion, results in the thinning of the tube. The strain in the tube can be written as

$$
\int \mathrm{d} \epsilon=-\int \frac{\mathrm{d} T}{T}
$$

where, $T=$ tube thickness. Applying the volume constancy relation i.e.

$$
\left(D_{0}^{2}-D_{i}^{2}\right) * L=\text { Constant ; }\left(D_{0}+D_{i}\right) *\left(D_{0}-D_{i}\right) * L=(\tilde{D}) * 2 T * L=\text { Constant },
$$

where $D_{0}$ and $D_{i}$ are the outer and inner diameters of the tube and $\tilde{D}=\left(D_{0}+D_{i}\right) / 2$ is the average diameter of the tube. Ignoring the axial change in length $\tilde{D} T=K$ (constant) ( $\tilde{D}=$ average tube diameter), from which it follows that $T \partial \tilde{D}=-\tilde{D} \partial T$. Using the above equations one obtains the following expression,

$$
\partial T=-\frac{K}{\tilde{D}^{2}} \partial \tilde{D}
$$

Using the average diameter the overall strain can be written as follows

$$
\epsilon=\int_{D_{1}}^{D_{2}} \frac{\partial \tilde{D}}{\tilde{D}}=\ln \frac{D_{2}}{D_{1}}
$$

where $D_{1}=$ initial average diameter; $D_{2}=$ current average diameter. The strain rate can be expressed as $\dot{\epsilon}=\frac{1}{\tilde{D}} \frac{\partial \tilde{D}}{\partial t}$.

From Lal and Hillier [17], the relationship of the balance of forces causing radial momentum in the tube would provide the expression for the resultant tangential stress $\sigma_{\theta}$,

$$
\begin{gathered}
\sigma_{\theta}=\frac{D}{2 T}\left\{\kappa P_{G e n}-(\rho T) \frac{\mathrm{d}^{2} D}{\mathrm{~d} t^{2}}\right\} \\
B_{0}=\mu_{0} \frac{N I}{l} \Gamma(\alpha, \beta), \Gamma(\alpha, \beta)=\frac{\beta}{\alpha-1} \ln \frac{\alpha+\sqrt{\alpha^{2}+\beta^{2}}}{1+\sqrt{1+\beta^{2}}}, \beta=\frac{l}{2 r_{1}}, \alpha=\frac{r_{2}}{r_{1}} \\
\kappa=\tau\{\Gamma(\alpha, \beta)\}^{2}
\end{gathered}
$$

Here $P_{G e n}$ is the generated magnetic pressure, $\rho$ is the mass density, $t$ is the time; $\kappa=\{0.1-0.167\}, r_{2}$ is outer radius of coil, $r_{1}$ is inner radius of coil, $l$ is active length of coil, $\Gamma(\alpha, \beta)$ is geometry dependent factor, $\tau$ is coupling dependent factor between coil and tube, and $\alpha, \beta$ are factors given by $6(\mathrm{~b})$.

It is to be noted that the above expression becomes identical to the one used by Janiszewski [18] to compute the tangential stresses for explosive expansion of ring during its deacceleration stage when generated magnetic pressure $P_{G e n}$ is negligible. The above expression is derived by ignoring the axial inertial forces. 
The thickness during expansion can be calculated from the volume constancy condition and by assuming that change in length is negligible during the expansion of the tube, hence $T=\frac{D_{o} T_{o}}{D}$.

\section{Numerical Simulation}

The use of electromagnetic numerical simulation helps to get an insight into the magnetic field distribution between coil and tube during electromagnetic forming [19]. In this work, a 2D axisymmetric simulation model is developed using COMSOL. Magnetic vector potential method is used to solve the Maxwell's equations [20] (see Table 3).

The following assumptions are made in the developed model [5].

- Displacement current and free charge density are neglected.

- The electrical conductivity and permeability of material are constant and isotropic.

- The effect of temperature on material properties is ignored.

\section{Results and Discussion}

\subsection{High Strain Rate Mechanical Properties}

The flow stress behaviour of annealed AA5052 at two selected strain rates between $1200-3000 \mathrm{~s}^{-1}$ is shown in Figure 5. The flow stress behaviour was found to be very similar at the two strain rates. The room temperature yield stress was about $150 \mathrm{MPa}$ that hardened to about $300 \mathrm{MPa}$ at a strain of 0.2 . It could be inferred from the nature of the curves that strain rate sensitivity of flow stress for AA5052 at room temperature and dynamic strain rates is about 0.09 , can be considered not very significant.

\subsection{EM Expansion of AA5052 Tubes}

Experiments as per Table 2 were performed. The photographs of the expanded tubes are shown in Figure 6. It is seen that up to a peak current of $131 \mathrm{kA}$ the tubes deformed but did not fracture. At peak currents of $140 \mathrm{kA}$ and higher the tubes fractured.

The diameter of expanded tubes was measured and the percentage change in diameter at different current level was determined as shown in Figure 7.

Table 3. Maxwell's equation in differential form.

\begin{tabular}{cc}
\hline Equation Name & Differential Form \\
\hline Maxwell-Ampere's Law & $\nabla \times H=J+\frac{\partial D}{\partial t}$ \\
Faradays Law & $\nabla \times E=-\frac{\partial B}{\partial t}$ \\
Guass's Law & $\nabla \cdot D=\rho$ \\
Guass's magnetic law & $\nabla \cdot B=0$
\end{tabular}




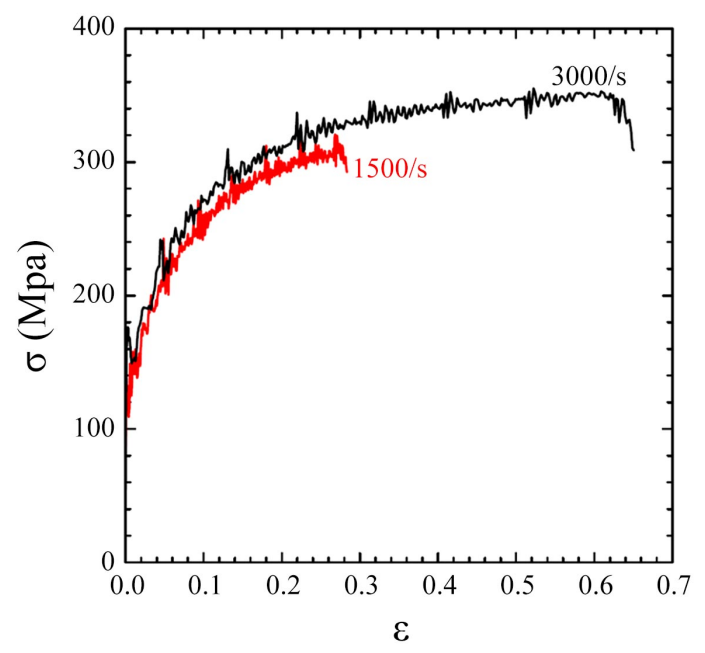

Figure 5. Stress strain curve of AA5052 at ambient temperatures and at two strain rates as indicated.

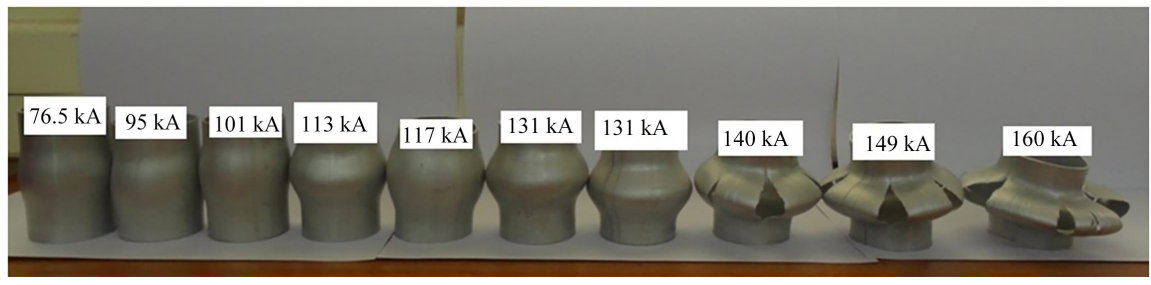

Figure 6. Expanded tubes after EMF experiments carried out at various current levels.

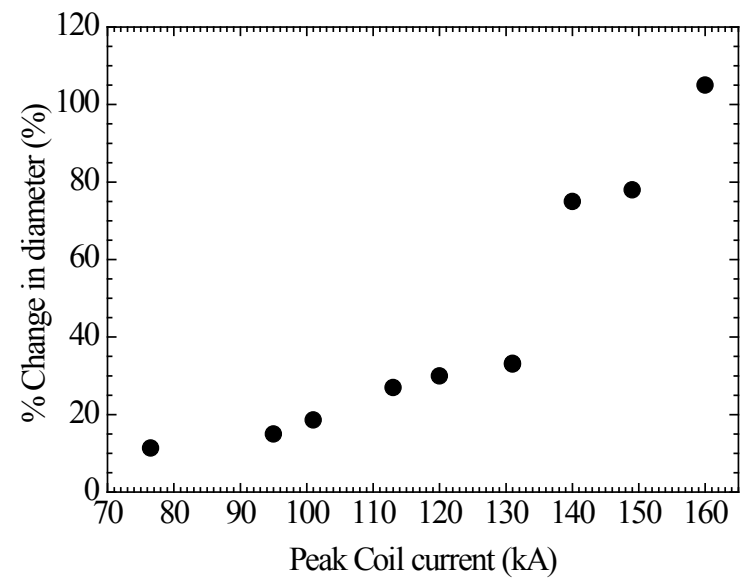

Figure 7. Percentage change in diameter of the EMF tubes at different peak currents.

\subsection{Simulation of Magnetic Pressure}

The stored electrostatic energy in capacitor bank is suddenly discharged into electromagnetic coil by closing the spark gap switches. The expression of current through the coil is given by Equation (1). The expression of generated magnetic pressure is derived in Equation (2). 2-D axis symmetric simulation was performed to calculate factor $\kappa$ see Equation (6c), which is a function of the coil geometry, gap between coil and tube, the tube material and the coupling be- 
tween coil and tube. Using the 2-D axis symmetric coil and tube arrangement (Figure 8(a) left image) the magnetic field at peak current around the coil was simulated (Figure 8(a) right map) where the magnetic field at tube surface at centre is plotted using equation $1 \mathrm{~b}$. Figure 8 (b) is simulation result at peak value of current $(117 \mathrm{kA}$ ) for 7 turns of solenoid coils. Along with supplied coil current (Figure 8(b)) the waveforms of $P_{G e n}$ as per Equation (2) is also shown, referred to as $P_{\text {calculated }}$. Superimposed on that is the pressure through simulation $P_{\text {simulation }}$. While $P$ simulated shows magnetic pressure at ID of tube surface. Figure 8 (b) shows that the calculated peak pressure is $200 \mathrm{MPa}$ and the simulated peak pressure is $60 \mathrm{MPa}$. From this $\kappa$ can be obtained as the ratio of the simulated to the calculated peak pressure, and is obtained as $\sim 0.33$. Figure 8 (c) shows the simulated radial displacement at the tube center as well as the estimated displacement rate from the displacement curve. It is seen that the time dependence of the radial displacement has a sigmoidal dependence. This nature of the curve has also been obtained during aluminium alloy tube expansion by electromagnetic forming where the radial displacement is monitored by Photonic Doppler Velocimetry (PDV) [21]. The displacement rate was estimated from the tube radial displacement-time curve using a seven point moving average of the slope at each recorded time data. The displacement rate shows a double hump separated approximately by quarter time period of the coil current cycle. This is similar to the direct measurement of velocity using PDV technique is A1060 alloy [22]. The simulation of displacement predicts a diametral expansion of $\sim 17 \mathrm{~mm}$. The maximum displacement rates are greater than $100 \mathrm{~m} / \mathrm{s}$. There is significant difference in the rates of the two peaks which is in variance with the observation by Jeanson et al. [22]. These differences could arise because of the simplifications used for simulations such as the use of $2 \mathrm{D}$ axi-symmetric model of tube, considering circular coils rather than helical and ignoring temperature dependency of the material.
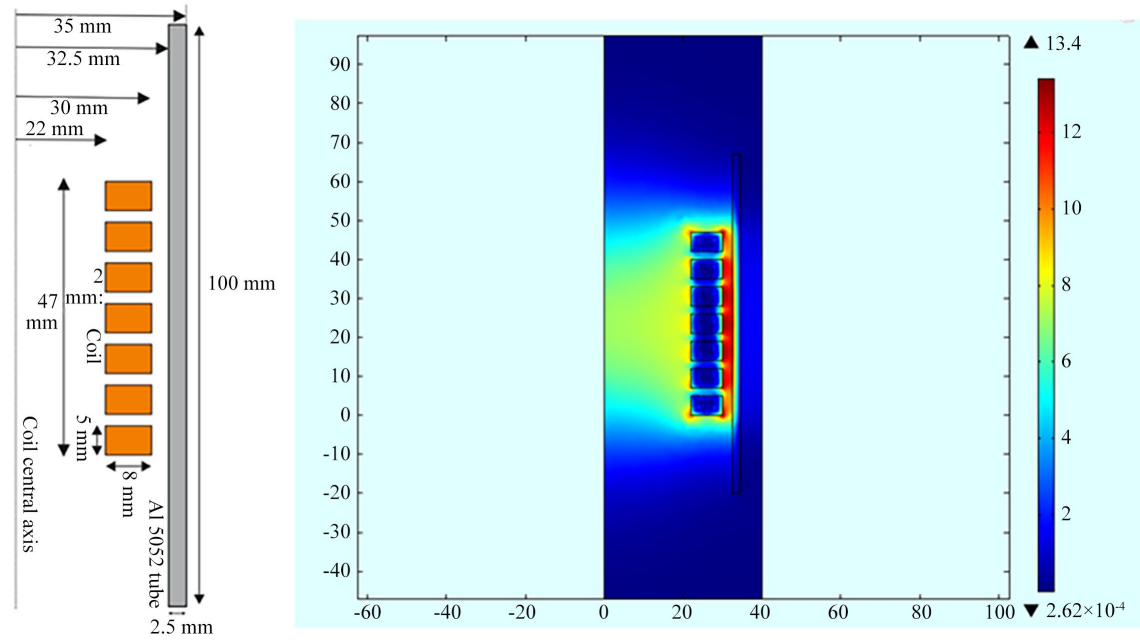

(a) 


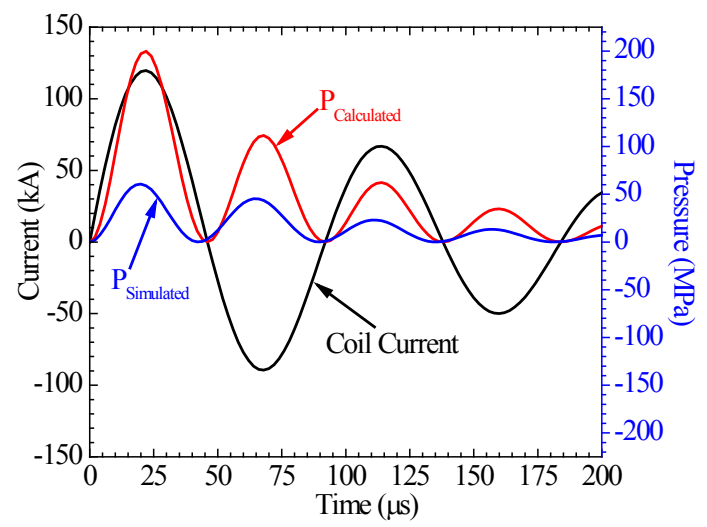

(b)

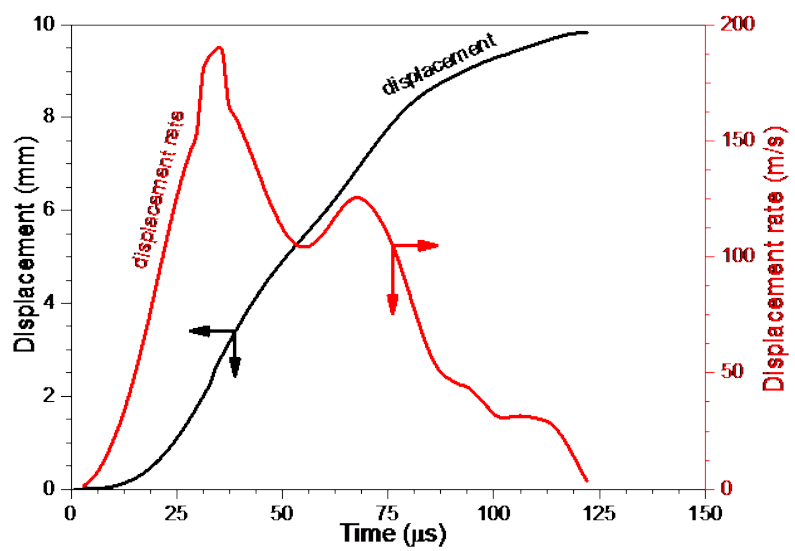

(c)

Figure 8. (a) 2-D axis symmetric coil and tube arrangement (left), Magnetic field distribution in Tesla at peak current (right) (b) Simulated result showing the calculated from Equation (2) and simulated magnetic pressure as well as the coil current. (c) Simulated displacement and deduced displacement rate at the tube centre.

\subsection{Visualisation of Imaging}

The expansion experiments with camera were carried out at two different levels of peak current at a charging level of $(15 \mathrm{kV}, 224 \mu \mathrm{F})$ and $(16 \mathrm{kV}, 112 \mu \mathrm{F})$, which resulted in a peak current of 117 and $140 \mathrm{kA}$, respectively. The lower level of 117 $\mathrm{kA}$ caused expansion of the aluminium tube and the higher current level of 140 kA led to not only expansion but also fragmentation of the tubes. The current waveform synchronized with camera is shown in Figure 9.

The sequence of images showing the tube expansion process under the action of peak current of $117 \mathrm{kA}$ and $140 \mathrm{kA}$ are shown in Figure 10. The time between two images was $98 \mu \mathrm{s}$ and is the sum of the exposure time $(60 \mu \mathrm{s})$ and read out time $(38 \mu \mathrm{s})$ of the camera. The expansion images were captured for $293 \mu \mathrm{s}$ for $117 \mathrm{kA}$ peak current and $488 \mu$ s for $140 \mathrm{kA}$ peak current within the given inter-frame rate. As pointed out that the camera voltage was synchronised with the minima of the current pulse, the expansion recorded is considered to be the average of the increasing and decreasing phases of the magnetic pressure on the specimen. During the rising portion of the current, the increasing magnetic 
pressure controls the expansion and in the decreasing portion, which represents weakening of the magnetic forces, the expansion is sustained by inertial forces. As in the subsequent pulse cycles, due to dampening of the peak current, there is progressive reduction of the contribution of magnetic pressure for expanding the tube. Thus, expansion is increasingly constrained after the first current cycle; as the developed magnetic pressure is unable to maintain the balance between the tangential stresses and inertial forces generated in the tube. This results not only in variation in the tangential stresses in the increasing and decreasing portion of the current cycle but also variation in the expansion rate of the tube and developed strain rate. In order to capture this behaviour the inter frame rate should be at least about 20 times faster than that used for the present work. Such information would be invaluable to elucidate the dynamics of the electromagnetic expansion process in materials. However for the purpose of the present work to characterise the broad stages in the expansion and fragmentation of tubes and to determine the nominal deformation parameters of stress, and strain rate the record of average increase in tube diameter extending both in acceleration and deacceleration stages is sufficient.

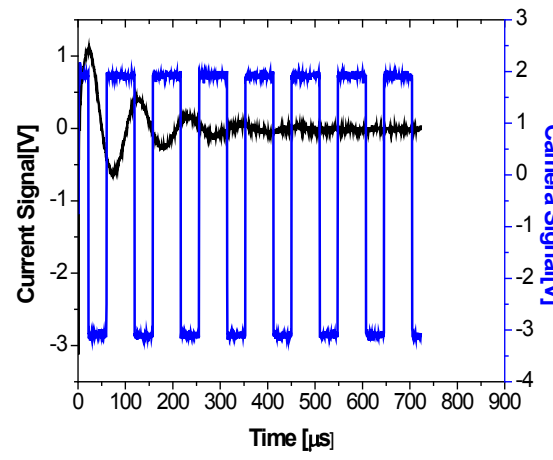

(a)

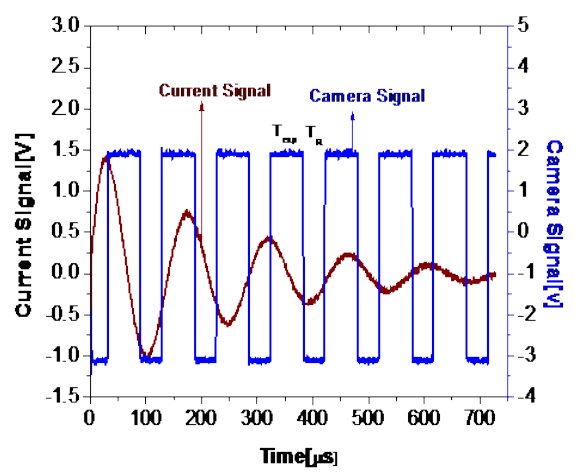

(b)

Figure 9. Current signal and camera signal with peak current of (a) $117 \mathrm{kA}$ and (b) 140 kA.

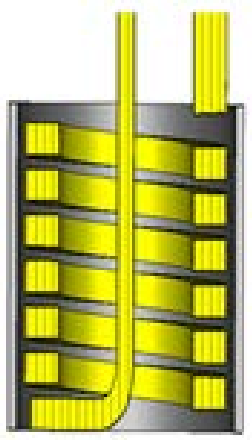

(a)
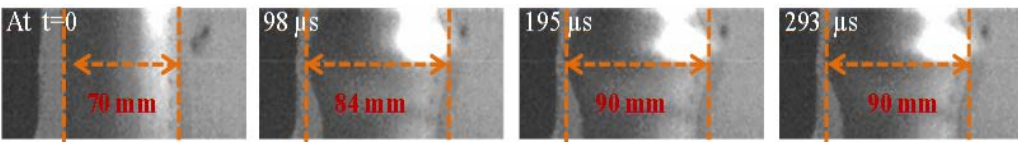

(b-i) 

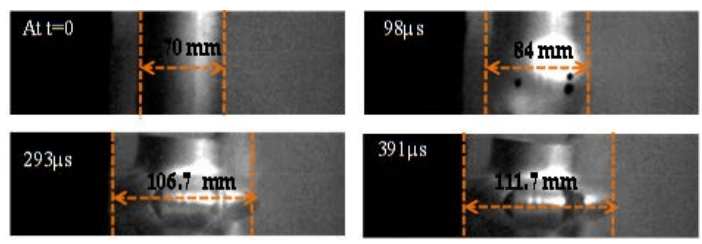

(b-ii)

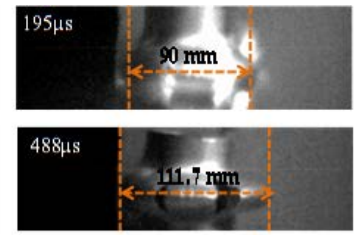

Figure 10. (a) Electromagnetic coil and tube arrangement. (b) Time snapshot image frames capturing expansion at peak current of (i) $117 \mathrm{kA}$ (ii) $140 \mathrm{kA}$.

\subsection{Estimation of Strain Evolution during Expansion}

The increase in diameter of the tube and its calculated thinning as a function of the test time for the two levels of peak current pulse used in the study is shown in Figure 11. The evolution of diameter $D$ was fitted with time $t$ to a third degree polynomial function as

$$
D=C_{o}+C_{1} t+C_{2} t^{2}+C_{3} t^{3}
$$

Similar approach was also adopted by Janiszewski [18] to describe the expansion of ring diameter by electromagnetic pulse. From the above equation describing the functional relationship between diameter and time of pulse one can derive the second derivative which is required for the computation of the tangential stresses,

$$
\frac{\partial^{2} D}{\partial t^{2}}=2 C_{2}+6 C_{3} t
$$

It is seen that the for the peak current of $117 \mathrm{kA}$ the diameter increases up-to $\sim 90 \mathrm{~mm}$ while for the peak current level of $140 \mathrm{kA}$ the diameter increases up-to $\sim 120 \mathrm{~mm}$. Thus it can be concluded that with increase in peak current greater expansion of the tube is obtained. From the images captured during expansion at the two peak current levels, it is seen that at $117 \mathrm{kA}$ the final expanded tube is intact, whereas the tube expanded at $140 \mathrm{kA}$ fragmented at $\mathrm{t} \sim 300 \mu \mathrm{s}$. It can thus be concluded that the fragmentation is initiated in the time range of 200 to 300 $\mu$ s after the tube has expanded within the range of 90 to $105 \mathrm{~mm}$. Figure 11(b) shows the variation of the estimated tube thickness assuming volume constancy. The final tube thickness estimated from the volume constancy relation is $\sim 2 \mathrm{~mm}$ for the tube expanded with peak current of $117 \mathrm{kA}$. The final thickness along the equatorial plane of the tube after expansion was measured with a wall thickness gauge to be on average $2.1 \mathrm{~mm}$ (the gauge is of Kroeplin make with a least count of $10 \mu \mathrm{m}$ ). Given the assumptions in calculations and the error in measurements and variation of tube thickness, the match is reasonable. In the tube expanded with a peak current of $140 \mathrm{kA}$ fragmentation occurred as a result of which the thickness could not be measured along the equatorial plane accurately. It is likely that the tube expansion and the concurrent thinning of the cross-section at the high rates could not be withstood by the tube leading to its fragmentation. To confirm this, the strain rate and the evolution of the generated forces and stresses in the tube need to be estimated in a reasonable manner. 


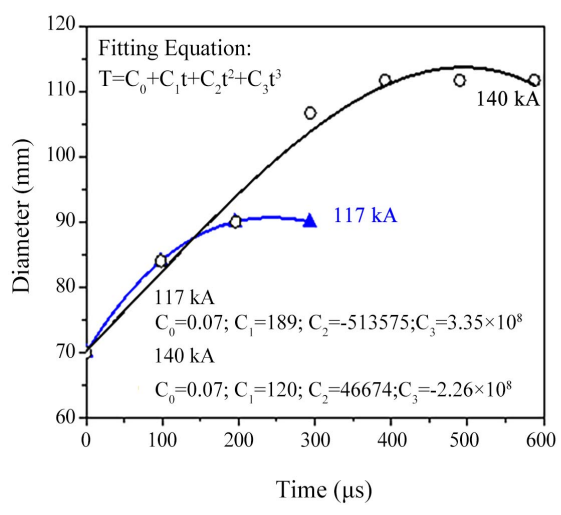

(a)

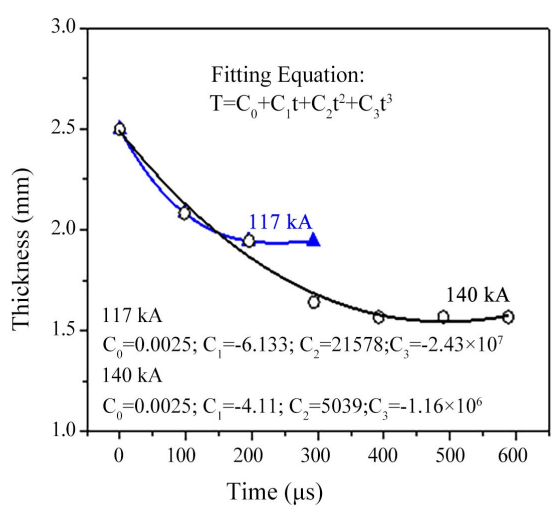

(b)

Figure 11. Evolution of (a) diameter and (b) thickness with time during EMF of AA5052. Also shown are the curves of third degree polynomial fitting the data.

\subsection{Estimation of Strain Rates}

The strain rates were calculated from camera images. In this calculation the spring back effect has been ignored as the effects on strain calculation is not considered significant. The strain rate is then simply the time elapsed for capturing the successive images using the fast camera. The evolution of the general diametral strain rate with strain for the two cases is shown in Figure 12. The abscissa shows strain calculated from Equation (5).

The plot in Figure 12 shows that in both the cases of expanding the tube at high and low peak current the starting strain rate is the same at $\sim 1750 \mathrm{~s}^{-1}$. As the imaging in both cases started at $95 \mu$ and showed similar diametral expansion values, the strain rate calculation turned out to be identical. In order to delineate the effect of the first generated pulse on the starting strain rate imposed much finer time scale of imaging at higher resolution is needed. Nevertheless the present set of experiments brings out the general trend in the evolution of the strain rate with strain as a result of expanding the tube at low and high peak current. It is found that for the lower peak current the strain rate rapidly decreases. In case the expansion of the tube is carried out at $140 \mathrm{kA}$ the strain rate drops to nearly a plateau value of an average of $1200 \mathrm{~s}^{-1}$, until fragmentation begins. From the images captured it is clear that the fragmentation occurred somewhere between 195 and $293 \mu$ s, which lies in the plateau region. The fragmentation leads to the superimposition of the axial rates over the diametral rates seen from a reduction of the overall tube length. Thus the onset of fragmentation results in decline in expansion along with sharp drop in the diametral strain rate. The extended strain plateau is due to the assumption that diametral thinning occurs till $293 \mu \mathrm{s}$ whereas it is clear that fragmentation did occur earlier. Nevertheless it is clear from the sequence of images of the tube expansion that the higher peak current of $140 \mathrm{kA}$ did increase the diameter to a larger extent than the peak current of $117 \mathrm{kA}$. This suggests that the magnetic forces generated by the higher peak current significantly aid in sustaining the expansion. 


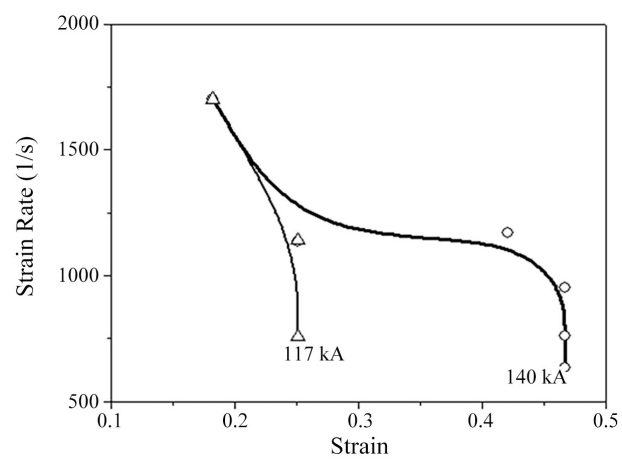

Figure 12. The plot of strain rate with strain during tube expansion for peak currents of 117 and $140 \mathrm{kA}$.

\subsection{Evolution of Tangential Stresses in Expanding Tube}

The variation of the magnetic force during the expansion process for the two peak currents used for expanding the tube is shown in Figure 13. In both the plots the band of yield stress of the material in the strain rate range imposed by the electromagnetic pulse is also shown. It facilitates determination whether the magnetic force generated due to the applied pulse is greater than the yield stress, and consequently induces plastic deformation of the material. It is seen that for the peak current level of $117 \mathrm{kA}$ only in the first pulse the generated peak force is greater than the yield stress of the material. In case of the peak current level of $140 \mathrm{kA}$ the first three pulses are within or above the yield stress. This indicates that by increasing the current level the generated peak magnetic force in increased in magnitude in the initial few pulses. As a result, the ability of the generated magnetic force to cause work-piece deformation is increased. Apart from the applied magnetic stresses which cause deformation only for fraction of the pulse when they are above the yield stress level, inertial stress generated in the deforming tube cause additional stress. The variation of the inertial stress during the test time is calculated from the peak current $117 \mathrm{kA}$.

The plot indicates that the inertial stresses remain above the band of yield stress until about $200 \mu \mathrm{s}$. For the case of peak current of $140 \mathrm{kA}$ the fragmentation of the work-piece hindered the estimation of the inertial stresses. However assuming conservatively that inertial levels are at least of the same levels as the peak current of $117 \mathrm{kA}$, it is clear that the total tangential stresses, by increasing the peak current to $140 \mathrm{kA}$, is significantly larger. The comparison of the tangential stresses generated in the work-piece estimated from Equation (6) is shown in Figure 14. As expected the tangential stresses are significantly larger for the peak current of $140 \mathrm{kA}$ as compared with $117 \mathrm{kA}$. They are not only larger in magnitude but also extend for longer duration above the yield level of the deforming work-piece material. The fragmentation of the tube at $140 \mathrm{kA}$ suggests that the stresses generated were not suitable for obtaining defect free expansion. Therefore the upper limit of expanding the tube of annealed AA5052 grade aluminium alloy is applying the electromagnetic pulse at $140 \mathrm{kA}$. 


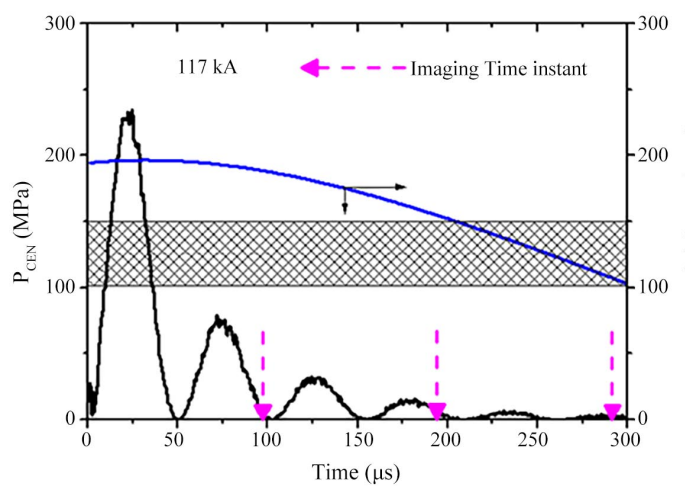

(a)

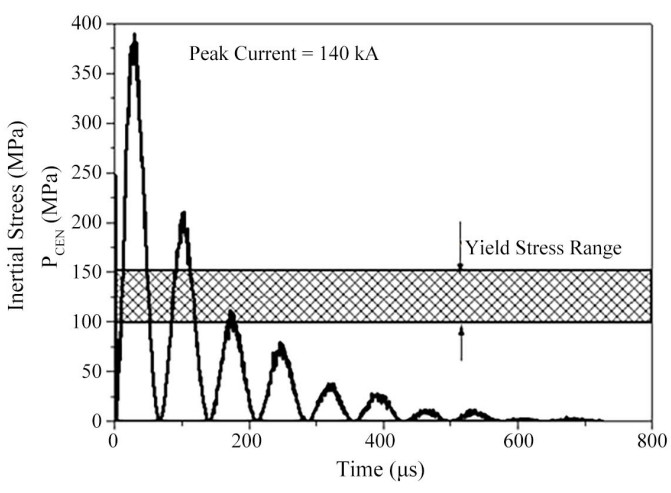

(b)

Figure 13. Plot showing the variation of magnetic pressure as calculated from Equation (1) for the peak current of (a) $117 \mathrm{kA}$ and (b) $140 \mathrm{kA}$. In plot (a) is shown the inertial stress calculated from Equation (6) (a) The band in both figures represents the yield stress range.

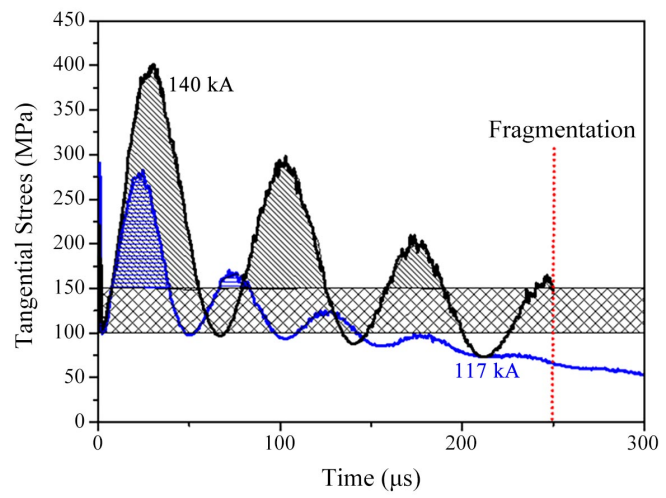

Figure 14. Plot comparing the estimated tangential stresses for the peak currents of 117 and $140 \mathrm{kA}$.

The present study on the expansion of aluminium tubes has systematically investigated the propensity for the tubes to remain intact over a wide range of current levels from $\sim 70 \mathrm{kA}$ to $140 \mathrm{kA}$. The analysis in the present study shows that strain rates of $\sim 1700 \mathrm{~s}^{-1}$ are developed resulting in peak displacement rates of $\sim 200 \mathrm{~m} / \mathrm{s}$ by using a novel imaging technique which synchronised imaging with the applied current. These values are consistent with the results obtained by Grady and Benson [23] for 1100 grade aluminium alloys obtained by high impact electromagnetic ring expansion test. However, in contrast to ring expansion, electromagnetic tube expansion provides in-plane deformations even at large strains under adiabatic conditions [22] which make the later more suitable for study of fragmentation. However the stresses are not uniaxial primarily because of axial inertial stress components creating a plane stress situation. Nevertheless the fragmentation is known to be governed by two broad factors-the capacity of the expanding material to absorb the impact occurring at high rates (the von karman velocity limit [24]) and the interplay of the radial and tangential inertial forces. Despite the complexity of the phenomenon a simplified analysis of the stresses in the expanding tube provides a qualitative understand- 
ing of the role of the tangential stresses before the onset of fragmentation. The subsequent manner of fragmentation observed in the study as unequal sections is consistent with the process governed by the release of Mott waves [25].

\section{Conclusions}

The following conclusions can be derived from the study.

1) Experiments have been conducted for current levels varying from $76 \mathrm{kA}$ to $160 \mathrm{kA}$ in 7 turns electromagnet.

2) The expansion of aluminium tube was imaged using high-speed camera synchronised with current which facilitated an estimation of the imposed strain rate and tangential stresses.

3) The expansion of the tube at peak current levels of $76.5 \mathrm{kA}$ to $131 \mathrm{kA}$ resulted in uniform expansion and that carried out using above $140 \mathrm{kA}$ resulted in fragmentation.

4) The strain rate of tube expansion in both the peak currents was in the range $1700-1200 \mathrm{~s}^{-1}$.

5) It was found that the magnitude of the peak current with specific coil turns is decisive in the development of the level and duration of tangential stresses to cause either expansion or fragmentation of the expanding tube.

\section{Conflicts of Interest}

The authors declare no conflicts of interest regarding the publication of this paper.

\section{References}

[1] Lal, G.K. (1972) Electromagnetic Forming. IEEE Transaction on Industry Applications, 8, 425-429. https://doi.org/10.1109/TIA.1972.349832

[2] Psk, V., Risch, D., Kinsey, B.L., Tekkaya, A.E. and Kliener, M. (2011) Elctromagnetic Forming-A Review. Journal of Materials Processing Technology, 211,787-829. https://doi.org/10.1016/j.jmatprotec.2010.12.012

[3] Guduru, P.R. and Freund, L.B. (2002) The Dynamics of Multiple Neck Formation and Fragmentation in High Rate Extension of Ductile Materials. International Journal of Solids and Structures, 39, 5615-5632. https://doi.org/10.1016/S0020-7683(02)00367-0

[4] Mamalis, A.G., Manolakos, D.E., Kladas, A.G. and Koumoutsos, A.K. (2004) Physical Principles of Electromagnetic Forming Process: A Constitutive Finite Element Model. Journal of Materials Processing Technology, 161, 294-299. https://doi.org/10.1016/j.jmatprotec.2004.07.039

[5] Lee, S.H. and Lee, D.N. (1996) Estimation of the Magnetic Pressure in Tube Expansion by Electromagnetic Forming. Journal of Materials Processing Technology, 57, 311-315. https://doi.org/10.1016/0924-0136(95)02086-1

[6] Qiu, L., Yu, Y.J., Xiong, Q., Deng, C.Z., Cao, Q.L., Han, X.T. and Li, L. (2018) Analysis of Electromagnetic Force and Deformation Behavior in Electromagnetic Tube Expansion With Concave Coil Based on Finite Element Method. IEEE Transactions on Applied Superconductivity, 28, 1-5. 
https://doi.org/10.1109/TASC.2017.2789287

[7] Psyk, V., Gerstein, G., Barlage, B., Albuja, B., Gies, S., Tekkaya, A. E. Bach, F.-W. (2012) Process Model and Design for Magnetic Pulse Welding by Tube Expansion. 5th International Conference on High Speed Forming, 24-26 April 2012, Dortmund.

[8] Thomas, J.D. and Triantafyllidis, N. (2007) Theory of Necking Localization in Unconstrained Electromagnetic Expansion of thin sheets. International Journal of Solids and Structures. 44, 6744-6767. https://doi.org/10.1016/j.ijsolstr.2007.03.007

[9] Taylor, G. (1948) The Use of Flat Ended Projectiles for Determining Dynamic Yield Stress-I. Theoretical Considerations. Proceedings of the Royal Society of London. Series A, 194, 289-299. https://doi.org/10.1098/rspa.1948.0081

[10] Assay, J. (1997) The Use of Shock Structure Methods for Evaluating High Pressure Material Properties. International Journal of Impact Engineering, 20, 27-61. https://doi.org/10.1016/S0734-743X(97)87478-7

[11] Chen, W.W. and Song, B. (2010) Split Hopkinson Bar (Kolsky): Design Testing and Applications. Springer, New York.

[12] Ghorui, S., Tiwari, N., Meher, K.C., Jan, A., Bhat, A. and Sahasrabudhe, S.N. (2015) Direct Probing of Anode Arc Root Dynamics and Voltage Instability in a Dc Non-Transferred Plasma Jet. Plasma Sources Science and Technology, 24, Article ID: 065003. https://doi.org/10.1088/0963-0252/24/6/065003

[13] Tiwari, N., Sahasrabudhe, S.N., Tak, A.K., Barve, D.N. and Das, A.K. (2012) Investigation of Some Aspects of the Spray Process in a Single Wire Arc Plasma Spray System Using High Speed Camera. Review of Scientific Instruments, 83, Article ID: 025110. https://doi.org/10.1063/1.3675887

[14] Watanabe, M. and Kumai, S. (2009) High Speed Deformation and Collision Behaviour of Pure Aluminium Plates in Magnetic Pulse Welding. Materials Transactions, 50, 2035-2042. https://doi.org/10.2320/matertrans.L-M2009816

[15] Nemat-Nasser, S. (1985) High Strain Rate Compression Testing. In: Kuhn, H. and Medlin, D., Eds., ASM Handbook Vol. 8: Mechanical Testing and Evaluation, West Conshohocken, ASM International, 190.

[16] Sharma, S., Kapoor, R., Chavan, V.M., Agrawal, R.G., Patel, R. J. and Chakravartty, J.K. (2011) Split-Hopkinson Pressure Bar: An Experimental Technique for High Strain Rate Tests, BARC Mumbai, India External Report, BARC/2011/E/013.

[17] Lal, G.K. and Hillier, M.J. (1969) The Expansion of a Thin Free Tube in Electromagnetic Forming. International Journal of Production Research. 8, 59-64. https://doi.org/10.1080/00207547008929829

[18] Janiszewski, J. (2012) Measurement Procedure of Ring Motion with the Use of High Speed Camera during Electromagnetic Expansion. Metrology and Measurement Systems, 19, 797-804.

[19] Giannoglou, A., Kladas, A., Tegopoulos, J., Koumoutsos, A., Manolakos, D. and Mamalis, A. (2004) Electromagnetic Forming: A Coupled Numerical Electromagnetic Mechanical Electrical Approach Compared to Measurements. Compel, 23, 789-799. https://doi.org/10.1108/03321640410540737

[20] Noh, H.G., Song, W.J., Kang, B.S. and Kim, J. (2015) Two-Step Electromagnetic Forming Process Using Spiral Forming Coils to Deform Sheet Metal in a Middle-Block Die. The International Journal of Advanced Manufacturing Technology, 76, 1691-1170. https://doi.org/10.1007/s00170-014-6392-4

[21] Thomas, J.D., Triantafydiliys, N., Vivek, A., Dhaen, G.S. and Bradley, J.R. (2010) 
Comparison of Fully Coupled Modelling and Experiments for Electromagnetic Forming Process in Finitely Strained Solids. International Journal of Fracture, 163, 67-83. https://doi.org/10.1007/s10704-010-9473-x

[22] Jeanson, A.-C., et al. (2016) A Coupled Numerical Experimental Approach for Characterisation of Material Behaviour at High Strain Rate Using Electromagnetic Tube Expansion Testing. International Journal of Impact Engineering, 98, 75-87.

[23] Grady, D.E. and Benson, D.A. (1983) Fragmentation of Metal Rings by Electromagnetic Loading. Experimental Mechanics, 23, 393-400. https://doi.org/10.1007/BF02330054

[24] Von Karman, T. and Duwez, P. (1950) The Propagation of Plastic Deformation in Solids. Journal of Applied Physics, 21, 987. https://doi.org/10.1063/1.1699544

[25] Mott, N.F. (1947) Fragmentation of Shell Cases. Proceedings of the Royal Society of London. Series A, 189, 300-308. https://doi.org/10.1098/rspa.1947.0042 